\author{
Journal of Reseanch of the National Burcau of Standards \\ Volume 91, Number 2, March-April 1986
}

\title{
Environmental Specimen Banking \\ The Selection, Collection, Transport, and Storage or Biomedical Samples
}

\author{
F. H. Kemper and N. P. Luepke \\ University of Muenster, D-4400 Muenster, Federal Republic of Germany
}

Accepted: October 24, 1985

\begin{abstract}
In order to adequately ensure the protection of human health and the environment from the thousands of presently suspected hazardous substances and the new compounds added to those by new industrial processes, sophisticated approaches to hazard assessment and monitoring are being established. Environmental specimen banking (ESB) is necessary: useful, and important for environmental monitoring currently, and in the future for monitoring the past. ESB has already proved a good tool for recording inorganic and/or organic pollution trends over the years. Moreover, ESB offers the possibilities and potentials for retrospective analysis of authentic samples from the past by improved future analytical procedures, including the detection of presently unnoticed environmental chemicals of biological interest. Among the specimens representing the environment, specimens of human origin play a key role. The selection criteria for human specimens include ethical and legal considerations together with the appropriate scientific approaches and epidemiological criteria. Technical considerations for sampling, preparation, transportation, and storage of the specimens include the selection and development of specific materials and implements, cold storage, and clean room technology in order not to compromise the original composition of the sample.
\end{abstract}

Key words: biomonitoring; environmental monitoring; environmental specimen banking; human specimens; monitoring human exposure.

\section{Introduction}

Ever since the industrial revolution, man has been subjecting the earth's biosphere to an increasing variety of chemical insults. Some, such as naturally-occurring toxic elements and compounds, are reentering the environment via industrial processes at rates much greater than their natural degradation or removal from the

About the Authors: F. H. Kemper, dean of the University of Muenster's faculty of medicine, is also director of the university's Institute of Pharmacology and Toxicology in which N. P. Luepke heads the Department of Environmental Toxicology. The work they report on is supported by the Federal Ministry of Interior and the Federal Ministry for Research and Technology and is coordinated by the Federal Environmental Agency of the Federal Republic of Germany. biosphere; other hazardous pollutants are being synthesized that have molecular structures never before encountered by living organisms. During the last decade, however, increasing attention has been focused on the occurrence and effects of pollutants in the environmont. Refinements in analytical techniques have resulted in the discovery that many substances are geographically more widely distributed than previously assumed. Serious accidents with exposure to and accumulation of toxic materials have lead to a public demand for more effective governmental control.

A list of all presently suspected environmental hazardous substances would contain thousands of chemical compounds; and industry is adding new compounds to that list every year. Up to now we are not in the position to analyze environmental samples for all 70,000 or so man-made chemicals and their metabolites circulating in the environment today. In order to adequately ensure the protection of human health and the 
environment from the often subtle effects of these materials, it is necessary to perform several major activities. These include toxicological research, control technology development, the promulgation of regulatory guidelines and standards and monitoring of environmental materials and specimen banking. There are at least three vital services which a biological specimen bank can render:

1) Provide up-to-date information on the spread of man-made chemicals in the environment, including man;

2) Allow extrapolation of concentration trends with respect to chemicals considered to present threats to man and his environment; and

3) "Preserve the presencc" by "fossilizing" selected environmental specimens in a repository so as to be able to analyze them for specific chemicals scavenged in past years.

These major activities are extremely important because without an effective monitoring of environmental materials and specimen banking the detection of serious environmental contamination from pollutants may occur after critical damage has been done.

Numerous environmental pollutants must be classified as health hazards for man; c.g., domestic poisons (detergents, pigments, propellants), naturally occurring poisons (plant or animal toxins), food components (additives, bacterial toxins, nitrosamines), stimulants (nicotine, ethanol, drugs). Human populations are increasingly exposed to substances that are potentially detrimental to health. Many of these substances are alrcady found in human tissues, fluids, secreta, and excreta, and some tend to concentrate in specific parts of the human body.

Biological monitoring programs can provide direct evidence of exposure and must therefore be implemented to supplement physical and chemical monitoring programs in areas where these are already in existence. Specimen banking will be of great importance in analyzing trends in exposure to previously unrecognized harmful pollutants or pollutants for which current measurement techniques are inadequate. Biological monitoring and analysis of stored specimens can provide a warning for the initiation of remcdial measures before critical damage occurs.

From the toxicologist's and pharmacologist's point of view, it is of interest in connection with xenobiotics to look for the target organs, the possibility of accumulation, and the hazards of exposure. The results of analytical work in real time monitoring as well as long-term trend analysis are the basis for medical estimation of the ecotoxicological risk concerning man's life.

Moreover, possibilities of conversions of pollutants in different systems must be taken into consideration; these conversions may result in substances with increased or lowered toxic qualities, e.g., for mercury and organohalogenated hydrocarbons.

\section{Goals and Objectives of Environmental Specimen Banking}

At present the number and variety of environmental chemicals are such that a systematic and allencompassing determination of their ecotoxic bchavior and discernible effects is all but impossible. Neither is it feasible to account for their circulation and accumulation in the environment. Furthermore, it seems selfevident that the same must hold for their decomposition products and metabolites.

In order to obtain more realistic information about the thrcats posed to man and his cnvironment by environmental chemicals, however, it seems logical and highly desirable to consider the systematic and repetitive collection of such environmental specimens which are known to scavenge and accumulate hazardous man. made compounds from their respective environment. Systematic and repetitive analyses over time of comparable samples will yield three most important types of information with respect to environmental chemicals already recognized or believed to be harmful:

1) Real time information as to the distribution of manmade chemicals and perhaps some of their decomposition products in the environment;

2) Trends with respect to increasing threats posed by certain environmental chemicals believed to be deleterious to the environment, including man; and

3) The long-term preservation of aliquots of such samples which were originally analyzed for mapping out the present-day distribution of known harmful chemicals and interpreted to determine any trend as which may exist. The possibility of analyzing well preserved samples ("fossils of the past") when nced arises in the future for the clarification of specific problems will help greatly to develop action plans as they will then be required and also to improve public policies.

Having recognized the potential importance of environmental specimen banking, the Government of the Federal Republic of Germany began developing plans for exploratory research late in 1974. An important impetus 
was provided by several ongoing R\&D activities in the U.S. which were initiated in 1971 and by German research proposals based upon these. Under U.S.-German Environmental Agreement of 1974, detailed plans began to take shape in 1975 for a joint R\&D effort. The German decision for funding nine selected projects was reached in January 1976. In its official Report on the Environment, dated 14 July 1976, the Government of the Federal Republic of Germany has specifically stated its intention to support the preparatory work toward establishing an environmental specimen bank (ESB). The German research program concentrates at present on the intake and metabolism of selected groups of environmental chemicals recognized to be harmful (e.g., group-fingerprint analyses with multiple apparatus linkups) and on basic research regarding sampling techniques, container materials, and storage techniques. The expansion of the German research efforts is currently being considered. The German environmental specimen bank pilotprogram is coordinated, developed, and cvaluated by the Federal Environmental Agency. Funds are provided by the Federal Ministry for Rescarch and Technology through an interagency agreement. Findings from a considerable number of $R \& D$ projects from the UFOPLAN (Environmental Research Plan), administered by the Federal Environmental Agency (some 500 current projects), as well as from pertinent R\&D activities sponsored by the Federal Ministry for Research and Technology, are also utilized.

The Environmental Specimen Bank and the parallel Data Bank, both situated at Muenster, started operation in mid-1980; a further bank was inaugurated in 1981 at Nuclear Research Center (KFA) Juelich.

\section{Selection of Specimens}

The scientific, logistical, and tcchnical problems associated with the development of selection criteria, biological sampling, and specimen banking programs arc generally recognized as being exceedingly profound and complex. Systems ecologists, toxicologists, physiologists, pathologists, molecular biologists, analytical chemists, and other participating scientists all have differing perspectives to bring to bear. Thus, the essential multidisciplinary nature of environmental monitoring and specimen banking will require maximum understanding, considerable forbearance, and some compromise among contributing disciplines.

Among the specimens representing the environment, specimens of human origin play a key role. Hence, the analytical results from these could be used for a critical (eco-)toxicological evaluation. Thus, the selection of human specimens should be made very carefully and should be ecologically meaningful with regard to the area of collecting samples.

According to the above-mentioned suggestions and in regard to the selection and characterization of a "best" human sample for monitoring and banking, the following summarized factors, some more obvious than others, should be considered in attempting to meet objectives:

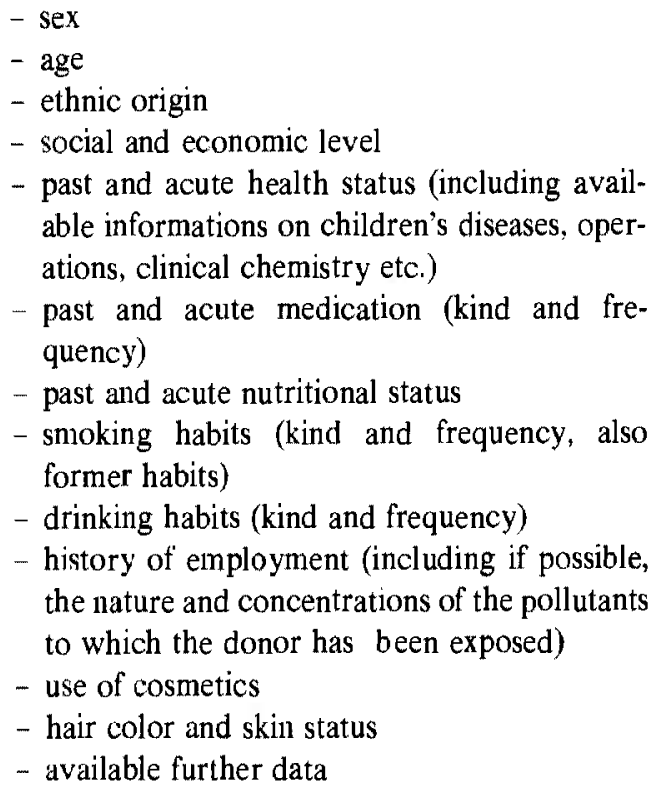
able informations on children's diseases, operations, clinical chemistry etc.)

- past and acute medication (kind and frequency)

- past and acute nutritional status

- smoking habits (kind and frequency, also former habits)

- drinking habits (kind and frequency)

- history of employment (including if possible, the nature and concentrations of the pollutants to which the donor has been exposed)

- use of cosmetics

- hair color and skin status

- available further data

There must be a differentiation between (parts of human organs to be obtained in real-time monitoring (RTM) and those for ESB. In RTM, whole blood (serum), urine, saliva, hair of different body regions, and nail material can be obtained, and if necessary and advisable, stored under appropriate conditions. In addition, for ESB, human organs are wanted that represent not only a momentary exposition but also long-term body burden of the analyzed chemicals. Materials for those specimens are available from autopsies and, incidentally, from surgical operations.

Joint working groups of the U.S. Environmental Protection Agency and the Federal (Republic of Germany) Environmental Agency have decided that suitable humanorgans of first priority for ESB are liver, adipose tissue, and whole blood. In addition, the storage of kidney, brain, and placenta was proposed. Within the pilot phase of ESB in the FRG, we collected and stored 19 different organs.

Generally it must be indicated that considerable thought must be given to establishing criteria for selection of biological specimens for biological specimen banking in aquatic and terrestrial ecosystems. Major considerations in selecting or recommending sensitive species or tissue sampling for biological specimen banking revolve around a set of broad biological problems including but not limited to the following points: 
- importance to man and environment

- position in the food chain

- availability and collection costs

An important fact for the choice of different kinds of mussels is their worldwide availability and the extensive experiences regarding bivalves as an indicator of water quality. The carp and the buttfish can be used as a predicator for the constitution of sediments and water. This is important as long as sediments are not tested dircetly also. The macroalgae are interesting because of their ability to accumulate heavy metals. The sludge was chosen because it is a very important indicator for industrial and urban pollution. A German basic research study found no great differcnecs in the composition of sludges taken from various areas.

In the area of food chain, the combination wheal/soil is of the same priority as cow milk. Here it should be seen that wheat is spread all over the world as important corn and that the concurrent investigation of the soil can yield important information. Carabids and earthworms were sclected as known indicators for the quality of soils and the front soil-atmosphere. In the area of air quality, the grass cultivation should get the first priority. For many years extensive experiences have been gained in Nordrhein-Westfalen about grass cultures as indicators of air pollution.

Because of the importance and the heterogeneity of samples of human origin, it was recommended that more than the average number of samples be taken from liver, blood, and fatty tissue. On the other hand, the number of samples taken from sludge can be limited because of the similar composition which is nearly independent of origin.

- distribution and abundance

- mobility and action radius

- accumulation of environmental pollutants

These factors and perhaps some further similar ecological considerations must be addressed as criteria along with the physical-chemical factors of collection methods, transportation, analysis, storage, and cost considerations.

In regard to these suggestions and the environmental specimen pilotbank the following bioindicators were discussed and taken in priority:

1) human tissues - liver, blood, fatty tissue

- kidney, brain, placenta

2) aquatic ecosystem

marine

- macroalgae

- Mytilus spec. Ostrea edulis, Pleuronectes fresh

- Dreissena polymorpha, Cyprinus carpio, sludge

3) tcrrestrial ecosystem

- wheat/soil, cow milk, Carabids, earthworm, grass culture.

The following considerations influenced these decisions: The liver has to be prefered to the kidney because of the size of organ and because of its homogeneity as shown by trace element distribution studies. The fatty tissue and the brain are interesting for the lipophilic compounds. Because of ethical and legal considerations the fatty tissue should be preferred. For the "Real-TimeMonitoring-Program," milk, whole blood, hair, skin, urine, are suggested because they are more available.

\section{Technical Considerations}

In the sampling, preparation, transportation, and storage of environmental materials and specimens for monitoring and bank programs, certain strict precautions must be taken to obtain adequate information relative to the purpose of the study and the substance to be measured. Analytical chemistry begins when a sample is taken; the analyst should be fully involved from this point. Contamination of samples and loss of volatile pollutants during collection and following steps may be more of a problem in some cases than others. Specific risks of contamination and loss are described in the following.

The wide spread of reported values for both organic and inorganic substances (especially in the $\mathrm{ng} / \mathrm{g}$ level) can be derived from uncritical application of analytical techniques as well as from "positive" or "negative" contamination.

Contamination and loss problems start with collection of environmental materials and biological specimens. Sampling in areas remote from laboratories requires certain strict precautions. Samples that have been subjected to prolonged transportation under adverse conditions may be useless and collection should therefore be restricted to those samples that can be adequately preserved in the field. Speed is of the essence if one wants to minimize the errors in estimates of pollutants within organisms that are obtained in the field. No chemicals may be used to collect environmental materials and specimens. Samples should be collected in an adequate, noncontaminating manner (e.g., shocking, trawling, and trapping).

Special cleaning procedures for materials and instruments used for sampling are necessary, as well as special sampling techniques to avoid contamination of the 
sample by pollutants from the environment during sampling environmental materials and specimens intended to be examined for inorganic pollutants. Similar consid-

' erations may be applied to knives, scissors, and other instruments necessary for organ dissection. Of course there are plastic or glass knives and scissors, but no guarantee is given that they do not contaminate the sample perhaps more than the metallic instruments. The recommended procedures for obtaining samples free from contamination include the use of quartz or borosilicate glass instruments, or those made of titanium, titanium carbide, boron carbide, carbon steel, or aluminium.

Titanium knives have been used with the result of no contamination except for titanium. They may be easier to use than the other types of instrument. Contamination by copper, manganese, chromium, nickel, iron, cobalt, silver, tin, antimony, and tantalum have been dcmonstrated by several authors when using stainless steel instruments. Recently the use of lasers has been proposed for cutting biological tissues, but laser cutting might produce excessive heat at the cut surface and potential losses of volatile organic and inorganic compounds or pyrolytic changes.

For organic compounds, instruments and other materials made of organic compounds that come into contact with the sample must be avoided (plastic gloves should be used with caution). Studies requiring data on the distribution of a substance within a tissue would, of course, involve the preservation of the intact tissue or whole organ. Homogenization of the spccimen may be employed in instances where a substance is inhomogeneously distributed or where replicate analyses are to be carried out by different labs. However, great care must be exercised with the homogenization procedures to avoid contamination.

All specimens should be transferred to permanent storage containers as soon as possible and handled as few times as possible bearing in mind that each additional handling enhances the possibility of introducing contamination.

For transport and storage, the container materials are also of great importance, e.g., to avoid migration from the specimen into the wall of the container or contamination from the container. There have been numerous studies performed in the past to find out the most suitable materials that will be inert and will neither lead to migration effects nor catalyze unwanted chemical reactions in the sample. It has been shown that glass (borosilicate glass) gives the needed safety. However, problems may arise due to a lack of reliable methods of sealing. For the estimation of inorganic compounds, plastic material may also be useful, but should be cleaned very carefully, e.g., with diluted acids prior to use. This is necessary because of the observation that inorganic residues of this material can be leached during this type of cleaning.

To preserve the integrity of samples, all possible types of storage were carefully investigated, resulting in the following conclusions:

1) Chemical preservation is not possible as most organic compounds are affected.

2) The possibilities of using irradiation are under investigation but seem not to be advantageous.

3) Lyophilization can obviously be used for those samples destined for the estimation of inorganic trace elements compounds, though the loss of highly volatile elements like mercury seems to be possible.

4) The storage of extracts may be an advantage only in atmospheric samples; further studies are necessary.

5) Ashing at low temperatures could probably be used for trace metal pollutants but will be limited to certain elements.

6) Rapid deep-freezing is the most acceptable procedure for maintaining sample integrity and preservation of contaminants.

It has been shown in our own investigations that deepfreezing provides good stability of the sample regarding both inorganic and organic contents (contaminants). Storage temperatures should be at $-80^{\circ} \mathrm{C}$ or lower. Thus, walk-in deep freezers, as well as liquid nitrogen freezers or containers, can be used and have been proven to be preferable for long-term storage. Storage under thcse conditions makes it necessary to limit the sample size in order to avoid "transport" effects within the sample. Also, the thawing process must be the subject of strict precautions.

Appropriate and uniform sampling and storage protocols must be used by all participants in the program. Moreover, all techniques used in the storage of the specimens must be uniform. The technique of cryogenic brittle-fracture appears to be most suitable for the pulverization of frozen tissue.

\section{Legal and Ethical Aspects}

Most of the scientific community seldom thinks about the legal aspects of the conduct or communication of science. Aside from issues of patent or publication rights, only the occasional scientist, even in a regulatory agency, becomes familiar with water law, conservation law, administrative law, or courtroom procedures pertaining to the uses and limits of science in formulating 
public policy. Yet, all humanity and human endeavor exist and function subject to governance by the law.

The law is a creature of its community, a creature of man-made protocols, and is itself quite admittedly unable to reach conclusions in any area other than law per se. Therefore, in the interaction of science and the law it is of paramount importance to understand that at any time and under any circumstances, science remains science-with all of its own community standards of self evaluation and judgment-and the law too remains a system into itself. Thus law cannot make good science bad, nor bad science good. Therefore, the scientist need not fear it and, in fact, need only look to the law for governance in nonscientific aspects of scientific work.

We in science from time to time have had to consider the legal aspects of field and laboratory studies as they have been used for regulatory purposes. The information that we offer here represents not legal counsel but rathcr relates to the experiences of scientists helping to formulate public policy or to enforce existing law, and offers some afterthoughts about them.

There are differences among various countries and sometimes between states within the same country, with respect to the regulations, customs, and restrictions that must be considered in planning and implementing programs for the collection, storage, and analysis of biological specimens. One must especially distinguish between human and other biological specimens.

Experience with Western European and North American countries participating in the studies of the Organization for Economic Cooperation and Development has indicated that, with respect to data obtained from wildlife sampling and analysis could be limited by laws protecting wildlife, but difficulties arising from such laws are unlikely in view of the need to choose species which are abundant. However, there may be restrictions (e.g., closed seasons) on the capture of some bird or fish species.

Storage of specimens within the country of origin should not present difficulties, but any requirements to transport specimens across frontiers for analysis or specimen banking may infringe on laws of import and export or on disease control enforcement.

A broad review of the regulations, customs, and restrictions that must be considered in planning and implementing programs for the collection, storage, and analysis of nonhuman biological specimens is given in the proceedings of the International Workshop on Monitoring Environmental Materials and Specimen Banking, held in Berlin in 1978. During the International Workshop in the Use of Biological Specimens for the Assessment of Human Exposure to Environmental Pollutants, held in Luxembourg in 1977, a summary of legal and ethical aspects in respect to human biological specimens was given.
As mentioned above there are differences among various countries in this special field. For example in some countries regulations require a formal institutional committee review to ensure proper safeguards in the health of subjects, informed consent, and confidentiality of data. Each responsible investigator must be cognizant of and proceed according to the customs and legal restrictions applicable to his country and/or any collaborating nation. The investigator may find it desirable to seek legal advice before starting the program. It is generally accepted and should be recalled, above all, that the Nuremberg Code of 1947 precludes any experiment on a human subject without his "voluntary consent" and this principle has remained absolutely unchallenged. More recently the term "informed consent" has become widely used. Such consent for biological monitoring may be given, in particular, when there is a suspected exposure to environmental pollutants which may be detrimental to the health of the individual.

While the format of the "informed consent" to be used may vary from country to country, with the circumstances and the types of indicator specimens to be collected, the following basic elements of information should be considered for inclusion in "informed consent":

- procedure to be followed for taking the biolog. ical specimen

- description of any attendant discomforts and risks which might reasonably be expected for the individual and the community

- an assurance that the data and results will be kept confidential but that the individual (and/ or his physician) will be informed, if he/she wishes, of the results with comments regarding their significance.

- in the case of a study requiring repeated sampling, an instruction that the person is free to withdraw his consent and to discontinue participation at any time without prejudice to the subject.

In the case of collection of post mortem specimens, the concept of "informed consent" should be replaced by "permission of next-of-kin." This permission must be requested whenever appropriate and some information regarding the purpose of the study should be provided if requested. Usually permission to acquire post mortem specimens is more difficult to obtain than ante mortem specimens because of legal reasons, as well as local customs or mores. The need for collaborating pathologists to perform autopsies in the accordance with the laws of the country is emphasized. In conclusion, both science and law are evolving independent systems unto them- 
selves. They do not change each other in their practice, but only in their evolution of thought. Thus, the sampling, archiving, and utilization of biological tissues never shall affect the practice of law, but may influence the development of present and future legislation concerning environmental protection, natural resource management, conservation, preservation, and other human benefits. Similary: passage of hmman law cannot affect the laws of nature, but it can influence the activities of science and scientists by providing positive and negative incentives to investigate selected subjects.

\section{Conclusions and Recommendations}

Environmental specimen banking (ESB) is necessary, useful and important for environmental monitoring currently, and in the future for representing the past. ESB has already been proven to be a good tool for recording inorganic and/or organic pollution trends over the years. Moreover, ESB offers the possibilities and potentials for retrospective anal ysis of authentic samples from the past by improved future analytical procedures, including the detection of presently unnoticed environmental chemicals of biological interest. Besides this, ESB is also of interest in supervising the effectiven ss of restrictions, regulations, etc., because pollution problems are woridwide and include terrestrial, aquatic, and atmospheric contamination. The operation of ESB should be harmonized on an international basis.

In order to ensure the efficient and economical operation of specimen banks, sample collection, storage, analysis and quality control of data should be centrally located or at least coordinated for each program and the number of storage sites and analytical laboratories kept to a minimum.

The above activities should be harmonized internationally as much as possible. They should utilize the resources and experiences of closely related programs which already exist, e.g., the U.S.-German Environmental Tissue Bank Program, the U.S. Pesticide Monitoring Program and the Mussel Watch Program. All highly industrialized countries, and increasingly developing nations, too, have yet to come to grips with the complex problems of providing facts and figures for establishing ecological criteria as baselines for environmental impact assessments. This is as true for human installations and activities already impacting upon the ecosphere as it is for the prejudgment of the future ecological consequences of plans as yet to be implemented.

Major activities are necessary to adequately ensure the protection of human health and the environment from the adverse effects of environmental pollutants. These activities include toxicological and ecological research, control technology development, the promulgation of regulatory guidelines and standards, and the monitoring of environmental materials and specimen banking. In the absence of effective monitoring of environmental materials and specimen banking, the detection of serious environmental contamination from pollutants may occur only after critical damage has been done. The link between legal wants and scientific necessities must be found. Regulations must cover a maximum of safety and a minimum of scientific demands. Only in this way cast a practicable "environmencal protection" be established that considers both: man against environment and environment against man.

\section{References}

[1] Berlin, A., et al. (eds.) Use of Biological Specimens for the Assessment of Human Exposure to Enwironmental Pollutants, M. Nijhoff Publ., Den Haag. Netherlands (1978).

[2] Luepke, N.P. (ed.) Monitoring Environmental Materials and Specimen Banking, M. Nijhoff Publ., Den Haag, Netherlands (1979).

[3] Lewis, R. A., et a1. (eds.) Environmental Specimen Banking and Monitoring as Related to Banking, M. Nijhoff Publ., Der Haag, Netherlands (1984).

[4] Kemper, F. H., Experiences in Monitoring and Banking Human Biological Specimens, in 2 (1979).

[5] Luepke, N. P., and F. Schmidt-Bleek, State-of-the-Art of Biological Specimen Banking in the Federal Republic of Germany, in 2 (1979).

[6] Kemper, F. H., and N. P. Luepke Geperal Aspects of Monitoring and Banking of Human Biological Specimens, in 3 (1984). 